\title{
Update on clinical, imaging, and therapeutic aspects of kissing molars: A systematic review
}

\author{
Atualização sobre aspectos clínicos, de imagem e terapêuticos dos molares beijadores: uma revisão \\ sistemática
}

Actualización sobre los aspectos clínicos, de imagen y terapéuticos de besar los molares: una revisión sistemática

Received: 04/26/2021 | Reviewed: 05/05/2021 | Accept: 05/10/2021 | Published: 06/10/2021

\author{
Ícaro Girão Evangelista \\ ORCID: https://orcid.org/0000-0001-8728-6694 \\ Universidade Federal do Ceará, Brazil \\ E-mail: icarogirao8@gmail.com \\ Eduardo Costa Studart Soares \\ ORCID: https://orcid.org/0000-0002-7188-9308 \\ Universidade Federal do Ceará, Brazil \\ E-mail: estudart@yahoo.com.br \\ Daniel Almeida Ferreira Barbosa \\ ORCID: https://orcid.org/0000-0002-9557-5637 \\ Universidade Federal do Ceará, Brazil \\ E-mail: danielodontologia@gmail.com \\ José Nunes Carneiro Neto \\ ORCID: https://orcid.org/0000-0003-2388-7540 \\ Universidade Federal do Ceará, Brazil \\ E-mail: jnunes.neto@yahoo.com.br \\ Edson Luiz Cetira Filho \\ ORCID: https://orcid.org/0000-0002-5985-8955 \\ Universidade Federal do Ceará, Brazil \\ E-mail: edson.cetira@hotmail.com \\ Francisco Samuel Rodrigues Carvalho \\ ORCID: https://orcid.org/0000-0002-3142-1268 \\ Universidade Federal do Ceará, Brazil \\ E-mail: samuelcarvalho@ufc.br \\ Paulo Goberlânio de Barros Silva \\ ORCID: https://orcid.org/0000-0002-1513-9027 \\ Centro Universitário Christus, Brazil \\ E-mail: paulo_goberlanio@yahoo.com.br \\ Fábio Wildson Gurgel Costa \\ ORCID: https://orcid.org/0000-0002-3262-3347 \\ Universidade Federal do Ceará, Brazil \\ E-mail: fwildson@yahoo.com.br
}

\begin{abstract}
Introduction: The Kissing Molar (KM) is defined by two or more lower posterior teeth in contact with the occlusal surfaces and the roots in opposite directions. Purpose: This study aimed to review epidemiological, clinical, and imaging aspects related to Kissing Molars. Methods: A two-phase systematic review registered in the PROSPERO platform was performed. Search strategies were developed for specific databases (PubMed, Scopus, Web of Science, Scielo, and LILACS), including the gray literature (Open Grey and Google Scholar). The CARE guideline was followed to evaluate methodological aspects, and the Joanna Briggs Institute instrument (MAStARI) assessed the risk of bias. Statistical analysis considered a 5\% level of significance. Results: The initial search strategy resulted in 564 articles. From this total, 21 studies (case reports and case series) meet the eligibility criteria. The main aspects associated with $\mathrm{KMs}(\mathrm{n}=32)$ were laterality difference between males and females $(\mathrm{p}=0.028)$, report of mental retardation $(\mathrm{p}=0.023)$, the involvement of second $(\mathrm{p}=0.036)$ and third $(\mathrm{p}<0.001)$ molars, surgical intervention $(\mathrm{p}<0.001)$ associating ostectomy and odontossection $(\mathrm{p}=0.028)$, and low occurrence of postoperative complications $(\mathrm{p}=0.002)$. Conclusion: A low number of KMs were reported in the literature. They showed statistically significant differences regarding epidemiological, clinical, imaging, and therapeutic characteristics. Thus, dental surgeons would consider these aspects before the treatment of patients with KMs.
\end{abstract}

Keywords: Dental follicle; Kissing molars; Rosette formation. 


\section{Resumo}

Introdução: Os Molares Beijadores (MB) são definidos por dois ou mais dentes posteriores inferiores em contato com as superfícies oclusais e as raízes em direções opostas. Objetivo: Este estudo teve como objetivo revisar aspectos epidemiológicos, clínicos e de imagem relacionados aos Molares Beijadores. Métodos: Foi realizada uma revisão sistemática em duas fases registrada na plataforma PROSPERO. Estratégias de busca foram desenvolvidas para bases de dados específicas (PubMed, Scopus, Web of Science, Scielo e LILACS), incluindo a literatura cinza (Open Gray e Google Scholar). A diretriz CARE foi seguida para avaliar os aspectos metodológicos, e o instrumento Joanna Briggs Institute (MAStARI) avaliou o risco de viés. A análise estatística considerou um nível de significância de 5\%. Resultados: A estratégia de busca inicial resultou em 564 artigos. Desse total, 21 estudos (relatos de casos e séries de casos) atendem aos critérios de elegibilidade. Os principais aspectos associados aos MBs $(n=32)$ foram diferença de lateralidade entre homens e mulheres $(p=0,028)$, relato de retardo mental $(p=0,023)$, envolvimento do segundo $(p=$ 0,036) e terceiro $(\mathrm{p}<0,001)$ molares, intervenção cirúrgica $(\mathrm{p}<0,001)$ associando ostectomia e odontossecção ( $\mathrm{p}=$ 0,028) e baixa ocorrência de complicações pós-operatórias $(\mathrm{p}=0,002)$. Conclusão: Um baixo número de MBs foi relatado na literatura. Eles mostraram diferenças estatisticamente significativas em relação às características epidemiológicas, clínicas, de imagem e terapêuticas. Assim, o cirurgião-dentista consideraria esses aspectos antes do tratamento de pacientes com MB.
\end{abstract}

Palavras-chave: Folículo dentário; Molares beijadores; Formação de roseta.

\title{
Resumen
}

Introducción: El Besar los molares (BM) se define por dos o más dientes posteriores inferiores en contacto con las superficies oclusales y las raíces en direcciones opuestas.Objetivo: Este estudio tuvo como objetivo revisar aspectos epidemiológicos, clínicos y de imagen relacionados con Besar los molares. Métodos: Se realizó una revisión sistemática de dos fases registradas en la plataforma PROSPERO. Se desarrollaron estrategias de búsqueda para bases de datos específicas (PubMed, Scopus, Web of Science, Scielo y LILACS), incluida la literatura gris (Open Gray y Google Scholar). Se siguió la guía CARE para evaluar aspectos metodológicos y el instrumento del Instituto Joanna Briggs (MAStARI) evaluó el riesgo de sesgo. El análisis estadístico consideró un nivel de significancia del $5 \%$. Resultados: La estrategia de búsqueda inicial resultó en 564 artículos. De este total, 21 estudios (informes de casos y series de casos) cumplen los criterios de elegibilidad. Los principales aspectos asociados con los BM $(\mathrm{n}=32)$ fueron la diferencia de lateralidad entre hombres y mujeres $(\mathrm{p}=0.028)$, reporte de retraso mental $(\mathrm{p}=0.023)$, la participación de segundo $(\mathrm{p}=0.036)$ y tercero $(\mathrm{p}<0.001)$ molares, intervención quirúrgica $(\mathrm{p}<0,001)$ asociando ostectomía y odontosección $(\mathrm{p}=0,028)$, y baja ocurrencia de complicaciones postoperatorias $(\mathrm{p}=0,002)$. Conclusión: En la literatura se reportó un bajo número de BM. Mostraron diferencias estadísticamente significativas en cuanto a características epidemiológicas, clínicas, de imagen y terapéuticas. Por lo tanto, los cirujanos dentales considerarían estos aspectos antes del tratamiento de pacientes con BM.

Palabras clave: Folículo dentario; Besar los molares; Formación de rosetas.

\section{Introduction}

The kissing molar (KM) is a clinical and imaging condition defined by the presence of at least two lower posterior teeth showing contact with their occlusal surfaces and their roots in opposite directions that are usually impacted and wrapped in a single follicular space (Van Hoof, 1973; Menditti, et al., 2015; Zerener, et al., 2016). They were first described by Von Roof in 1973, in Holand. Robinson et al (1991) published the first case of bilateral KMs in a healthy patient. Also called Rosette Formation (RF), this dental arrangement represents an extremely unusual finding, possibly diagnosed on routine radiographs or when investigated for non-eruption of posterior teeth (Menditti, et al., 2015). Occasionally, the KM formation may be related to mucopolysaccharidosis, a metabolic disorder that causes the absence of breakdown enzymes of glycosaminoglycans (GAGs) being present in different body tissues (Menditti, et al., 2015; Zerener, et al., 2016).

The treatment of the KM can be divided into conservative (Barros, et al., 2018) or surgical (Gonzalez-Perez, et al., 2015) procedures. Early orthodontic treatment is an alternative method for the anomalous repositioning of KMs (Barros, et al., 2018). Surgical intervention for removal of KM is the main form of treatment reported in the literature (Barros, et al., 2018). The most frequently adopted technique is the performance of odontossection with transalveolar method under regional anesthesia. Eventually, general anesthesia is indicated for some cases of surgical removal of KM (Gonzalez-Perez, et al., 2015).

Since the knowledge of clinical, imaging, and therapeutic aspects of a specific condition is important when surgical 
intervention is required, the use of systematic reviews are valuable methods to summarize current scientific evidence. Therefore, this study aimed to investigate the main aspects of KMs through a systematic review design. To date, there is no registered systematic review focused on KMs

\section{Methodology}

\subsection{Protocol and registration}

The present methodological design followed the Preferred Reporting Items for Systematic Reviews and MetaAnalyzes (PRISMA) (Liberati, et al., 2009). The research protocol was registered in the International Prospective Register of Systematic Reviews (PROSPERO) (Albuquerque, et al., 2018) under protocol number CRD42018115151.

\subsection{Search information and search strategy}

A systematic review was performed to answer the following question: what are the main epidemiological, clinical, imaging, and therapeutic aspects related to KMs? This topic was elaborated using the PECOS strategy:

1. Population (P): patients with KMs.

2. Exposure (E): KMs.

3. Control (C): not applicable.

4. Outcomes (O): frequency of epidemiological, clinical, imaging, and therapeutic aspects.

5. Study design (S): case reports and case series,

Appropriate truncations and word combinations were selected and adapted for each database search, the following keywords are used "Molar", "Molar, Third", "dentigerous cyst", "mucopolysaccharidoses", "hyperplasia" and "kissing molars". Additional information on the search strategies is provided in Appendix A, which can be found in the supplemental data in the online version of this article. All references were manipulated in a reference management program (EndNote, Thomson Reuters, Philadelphia, PA, USA) and duplicate reports were removed.

\subsection{Eligibility criteria}

\subsubsection{Inclusion criteria}

Case reports and case series of KMs were included.

\subsubsection{Exclusion criteria}

The following articles were excluded: (1) literature reviews, clinical trials, editor's notes, pilot studies and in vitro studies were excluded; (2) epidemiological studies; and (3) studies not written in Latin (Roman) alphabet.

\subsection{Information sources}

To identify the studies to be included in this review, an electronic search in PubMed, Scopus, Web of Science, Scielo, and Latin American and Caribbean Latin American and Health Sciences Information Center (LILACS) databases was performed. A partial search in the gray literature was performed on Google Scholar and OpenGrey. The search was performed until September 2019 and updated in February 2020, without restrictions regarding publication year or language (Appendix A).

\subsection{Study selection}

The examiners were trained to apply the selection criteria of the studies that happened in two phases. Phase 1 involved the analysis of titles and abstracts of all articles obtained from the databases in order to select the studies to be read in full and, for the screening, the Rayyan QCRI web and mobile app for systematic reviews were used (Ouzzani, et al., 2016). Phase 2 
included the complete reading of the articles chosen in Phase 1 to assess eligibility by the established inclusion and exclusion criteria. Two authors (I. G. E. and J. N. C. N.) participated independently in both phases. In phase 2, an additional manual search in the references of the selected studies after the complete reading was carried out by two researchers with the objective of finding possible articles inadvertently omitted during the searches in the databases. In the two phases, any disagreement between the two researchers was solved by a third author (F.W.G.C.).

\subsection{Data collection process}

The studies that met the inclusion criteria were carefully analyzed by two authors (I. G. E. and J. N. C. N.) independently. A customized table was previously made with the following variables of interest in the study, and it was used for the data extraction and tabulation. If there was any event of disagreement, the article was discussed between the two authors and a third researcher (F. W. G. C.) until consensus was established.

\subsection{Data items}

Regarding the CARE guideline (https://www.care-statement.org/) to evaluate case reports, the items used to evaluate the articles were: Title (item 1); Keywords (item 2); Summary (items 3a to 3d); Introduction (item 4); Patient Information (5a to 5d); Clinical Findings (item 6); Timeline (item 7); Diagnostic Assessment (items 8a to 8d); Therapeutic Interventions (9a to 9d); Follow up and Outcomes (items 10a to 10d); Discussion (items 11a to 11d); Patient Perspective (item 12); and Informed Consent (item 13) (Riley, et al., 2017).

\subsection{Risk of bias in individual studies}

The risk of bias (RoB) was assessed independently by two review authors (I. G. E. and J. N. C. N.). Any disagreement between them over the risk of bias items were resolved through discussion with a third reviewer (F.W.G.C.). It was considered the Joanna Briggs Institute critical appraisal checklist for randomized controlled trials (Tufanaru, et al., 2017), as follows: 1) Were patient's demographic characteristics clearly described?; 2) Was the patient's history clearly described and presented as a timeline?; 3) Was the current clinical condition of the patient on presentation clearly described?; 4) Were diagnostic tests or assessment methods and the results clearly described?; 5) Was the intervention(s) or treatment procedure(s) clearly described?; 6) Was the post-intervention clinical condition clearly described?; 7) Were adverse events (harms) or unanticipated events identified and described?; 8) Does the case report provide takeaway lessons?

The included articles were classified as the risk of bias and methodological quality using the Meta-Analysis of Assessment and Review Instrument (MAStARI) (Aromataris \& Munn, 2017). About the RoB, a percentage system based on other studies with similar methodologies ranging from "high"(studies with a "yes" number of less than 49\%), "moderate" (50\% to $69 \%$ of "yes"), and "low" (studies with $70 \%$ or more of "yes") (Riley et al., 2017). The RevMan Software (Review Manager, version 5.3 software, Cochrane Collaboration, Copenhagen, Denmark) was used to generate the RoB summary with adaptation for the eight questions of MAStARI.

\subsection{Statistical analysis}

The statistical analysis was performed with data tabulated in Microsoft Excel® and exported to Statistical Package for Social Sciences (SPSS) version 20.0 for Windows $®$ in which the analyzes were performed with a confidence of $95 \%$. Absolute and percentage frequencies were compared by Fisher's exact test or Pearson's chi-square test.

Accordingly, the classification by Gulses et al (2012) categorizes molars in close occlusal contact that comprise the KMs. This classification does not cover all existing cases in the literature. Thus, we proposed an addition to the Gulses 
classification and a new classification relating the KMs based on their anatomical location, according to panoramic images or computed tomography to better guide the dental surgeon in choosing the treatment modality with the patient.

\section{Results}

\subsection{Study selection}

The present study was based on clinical data published in the literature and generated results that allowed an assessment of the epidemiological, clinical, and imaging characteristics of KM. Characteristics of this dental set were also observed, which had no existing classification in the literature. Given the careful data collection of published articles, KMs received a modified classification for better planning and treatment according to the patient in the present review.

The search strategy resulted in 564 articles. After reading the titles and summaries of these articles, the authors noticed that 34 of them were in more than one database, which means that they were duplicated. Then, 535 were excluded because they were not related to the subject. After reading the remaining 29 articles, 8 studies were excluded because they did not meet the eligibility criteria. Therefore, 21 articles were included for the data synthesis, and the results are represented in a search flowchart (Figure 1).

Figure 1. Flow-chart of the literature search and selection criteria. Adapted from PRISMA.

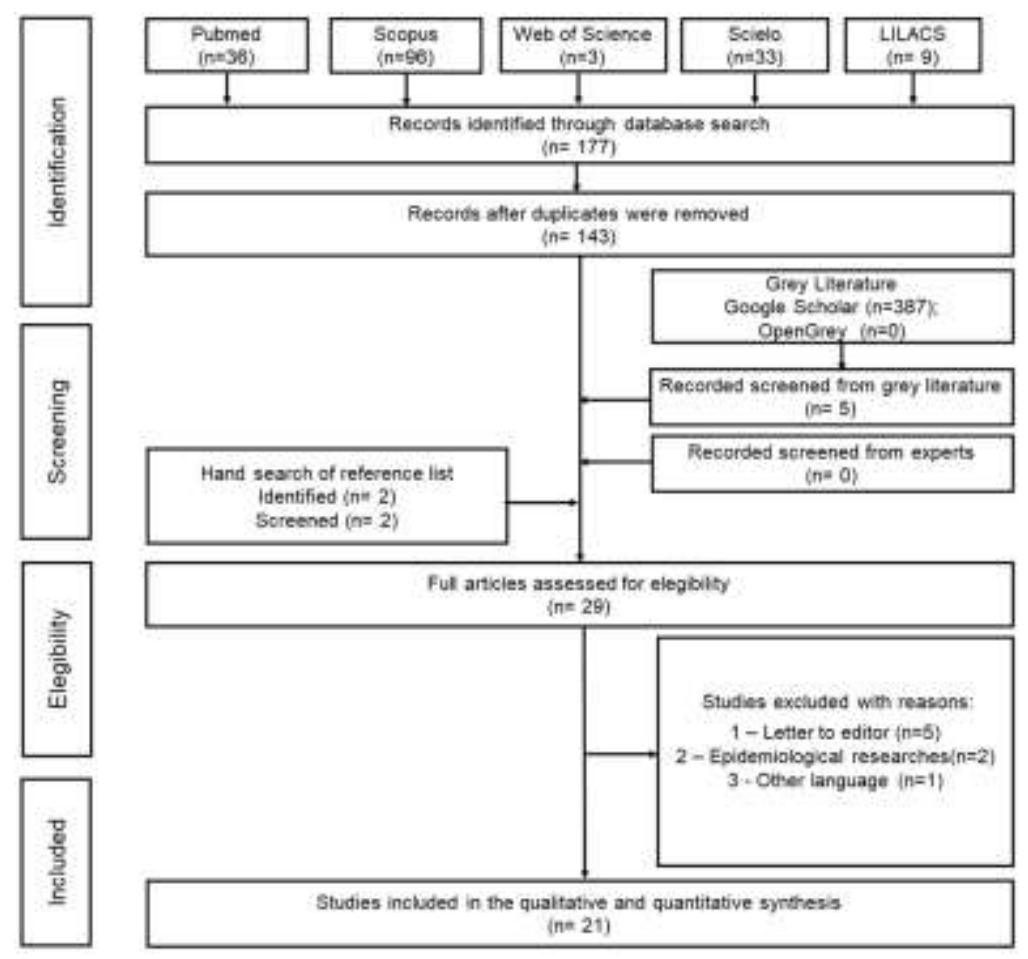

Source: Authors.

\subsection{Study characteristics}

The cases were distributed in the continents of South America $(n=4)$ (Barros, et al., 2018; Giraldi Neto, et al., 2012; Fortes, et al., 2014). Central America ( $\mathrm{n}=1)$ (Nuño \& Quijada, 2019) and North America ( $\mathrm{n}=1)$ (Robinson, et al., 1991), Asia (n=9) (Anish, et al., 2015; Cansiz, et al., 2016; Ghosh, et al., 2017; Jannu, et al., 2014; Kiran, et al., 2014; Nakamura, et al., 1992) and Europe (n=17) (Menditti, et al., 2015; Gonzalez-Perez, et al., 2015; Arjona-Amo, et al., 2016; Boffano \& Gallesio, 2009; McIntyre, 1997; Nedjat-Shokouhi \& Webb, 2014; Scheuber \& Bornstein, 2014). and. The distribution among countries was: 4 in Brazil (Barros, et al., 2018; Giraldi Neto, et al., 2012; Fortes, et al., 2014), 1 in the United States (Robinson, et al., 
1991), 1 in Mexico (Nuño \& Quijada, 2019), 4 in India (Anish, et al., 2015; Ghosh, et al., 2017; Jannu, et al., 2014; Kiran, et al., 2014), 2 in Turkey (Zerener, et al., 2016; Barros, et al., 2018), 3 in Japan (Nakamura, et al., 1992), 4 in Italy (Menditti, et al., 2015; Boffano \& Gallesio, 2009), 7 in Spain (Gonzalez-Perez, et al., 2015; Arjona-Amo, et al., 2016), 1 in Scotland (McIntyre, 1997), 1 in England (Nedjat-Shokouhi \& Webb, 2014), 1 in Switzerland (Scheuber \& Bornstein, 2014), 1 in the Netherlands (Van Hoof, 1973), (Figure 2). Bozkurt, et al (2017) published a series of 3 cases, but they did not describe the location of the study.

Figure 2. Distribution of the kissing molar cases among the continents.

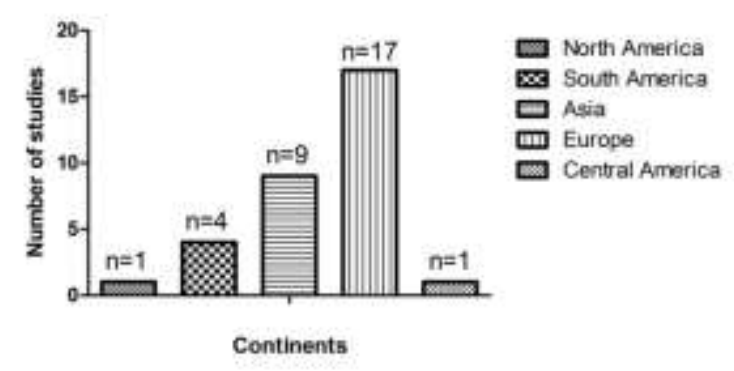

Source: Authors.

Most of the articles were published in English and an article was published in German comprising a total of 33 cases of KM reported, in which 18 cases are distributed in 6 series of cases (Menditti, et al., 2015; Gonzalez-Perez, et al., 2015; Giraldi Neto, et al., 2012; Nakamura, et al., 1992; Arjona-Amo, et al., 2016; Bozkurt, et al., 2017). Nakamura, et al (1992) and Arjona-Amo, et al (2016) reported 4 cases of KM each. Menditti, et al (2015), Bozkurt, et al (2017), and Gonzalez-Perez, et al (2015) described three cases in each of their articles. Giraldi Neto, et al (2012) published 1 article with 2 case reports, and the other authors reported only 1 case of KM in their respective works (Table 1). 
Table 1: Data on clinical findings, associated symptomatology, imaging and clinical presentation of kissing molars.

\begin{tabular}{|c|c|c|c|c|c|c|c|c|c|c|c|c|}
\hline \multirow[b]{2}{*}{ Author } & \multirow[b]{2}{*}{ Age(y) } & \multirow[b]{2}{*}{ Sex } & \multirow{2}{*}{\multicolumn{2}{|c|}{$\begin{array}{c}\text { Clinical } \\
\text { Signs }\end{array}$}} & \multirow[b]{2}{*}{$\begin{array}{l}\text { Metaboli } \\
\text { c Disturb }\end{array}$} & \multicolumn{2}{|c|}{ Clinical Symptom } & \multicolumn{2}{|c|}{$\begin{array}{l}\text { Imaginological } \\
\text { exams }\end{array}$} & \multicolumn{2}{|c|}{ Clinical presentation } & \multirow[b]{2}{*}{ Treatment } \\
\hline & & & & & & Pain & $\begin{array}{l}\text { Pericoronarit } \\
\text { is }\end{array}$ & PAN & CBCT & Bilateral & $\begin{array}{c}\text { Teeth } \\
\text { involved }\end{array}$ & \\
\hline $\begin{array}{l}\text { Soto NAI and } \\
\text { Soto QA, } 2019\end{array}$ & 32 & $\mathrm{~F}$ & I & + & - & + & + & + & - & - & $38 / 39$ & Surgical removal \\
\hline $\begin{array}{l}\text { Barros SE et } \\
\text { al, } 2018\end{array}$ & 10 & $\mathrm{~F}$ & I & + & - & + & + & + & - & - & $46 / 47$ & Orthdontics \\
\hline $\begin{array}{l}\text { Bozkurt P, } \\
2017\end{array}$ & 38 & $\mathrm{~F}$ & $\mathrm{NI}$ & - & - & - & - & + & + & - & $38 / 39$ & Surgical removal \\
\hline \multirow{6}{*}{$\begin{array}{l}\text { Ajona-Amo } M \\
\text { et al, } 2016\end{array}$} & 27 & M & NI & + & - & + & - & + & + & - & $37 / 38$ & Surgical removal \\
\hline & 22 & $\mathrm{~F}$ & NI & - & - & - & - & + & + & - & $48 / 49$ & Surgical removal \\
\hline & 35 & $\mathrm{M}$ & I & + & - & + & + & + & + & - & $47 / 48$ & Surgical removal \\
\hline & 24 & $\mathrm{~F}$ & I & + & - & + & - & + & - & - & $38 / 39$ & Surgical removal \\
\hline & 26 & $\mathrm{~F}$ & I & + & - & + & - & + & - & - & $47 / 48$ & Surgical removal \\
\hline & 31 & $\mathrm{~F}$ & II & + & - & + & - & + & - & - & $47 / 48$ & Surgical removal \\
\hline $\begin{array}{l}\text { Cansiz E et } \\
\text { al, } 2016\end{array}$ & 21 & $\mathrm{M}$ & II & - & - & - & - & + & - & - & $36 / 37$ & Surgical removal \\
\hline Gosh R, 2016 & 15 & $\mathrm{~F}$ & I & - & - & - & - & + & - & - & $37 / 38$ & $\begin{array}{l}\text { Surgical removal } \\
\text { and Orthdontics }\end{array}$ \\
\hline Zerener $T$, & 38 & $\mathrm{~F}$ & I & + & - & + & - & + & + & + & $37 / 38$ e $47 / 48$ & Surgical removal \\
\hline
\end{tabular}




\begin{tabular}{|c|c|c|c|c|c|c|c|c|c|c|c|c|}
\hline \multicolumn{13}{|l|}{2016} \\
\hline $\begin{array}{l}\text { Anish } N \text { et al, } \\
2015\end{array}$ & 35 & M & NI & - & - & + & - & + & - & + & $37 / 38$ e $47 / 48$ & NI \\
\hline $\begin{array}{l}\text { Gonzalez- } \\
\text { Perez LM et } \\
\text { al, } 2015\end{array}$ & 29 & $\mathrm{~F}$ & II & + & - & + & - & + & + & - & $47 / 48$ & Surgical removal \\
\hline \multirow{5}{*}{$\begin{array}{l}\text { Menditti D } \\
2015\end{array}$} & 34 & M & I & - & - & + & - & + & + & - & $47 / 48$ & Surgical removal \\
\hline & 29 & $\mathrm{~F}$ & I & - & - & + & - & + & - & - & $47 / 48$ & Surgical removal \\
\hline & 35 & $\mathrm{~F}$ & I & - & - & - & - & $\mathrm{NI}$ & $\mathrm{NI}$ & - & $36 / 37$ & Surgical removal \\
\hline & 56 & M & I & - & - & - & - & + & + & + & $38 / 39$ e $48 / 49$ & Surgical removal \\
\hline & 30 & $\mathrm{~F}$ & I & - & - & - & - & + & + & - & $38 / 39$ & Surgical removal \\
\hline $\begin{array}{l}\text { Jannu AA et } \\
\text { al, } 2014\end{array}$ & 18 & $\mathrm{~F}$ & I & - & - & - & - & + & - & - & $37 / 38$ & Surgical removal \\
\hline $\begin{array}{l}\text { Kiran HY et } \\
\text { al, } 2014\end{array}$ & 18 & $\mathrm{~F}$ & I & - & - & - & - & + & - & + & $37 / 38$ e $47 / 48$ & Surgical removal \\
\hline $\begin{array}{l}\text { Nedjatshokouh } \\
\text { i B and Webb } \\
\text { RM, } 2014\end{array}$ & 18 & M & I & - & - & - & - & + & - & + & $37 / 38$ e $47 / 48$ & Surgical removal \\
\hline $\begin{array}{l}\text { Sá Fortes RZ } \\
\text { et al, } 2014\end{array}$ & 33 & M & I & - & - & - & - & + & + & + & $37 / 38$ e $47 / 48$ & Surgical removal \\
\hline $\begin{array}{l}\text { Sheuber S and } \\
\text { Bornstein } M \text {, } \\
2014\end{array}$ & 48 & $\mathrm{~F}$ & $\mathrm{NI}$ & - & - & + & - & + & + & - & 47 e 48 & NI \\
\hline \multirow[t]{2}{*}{$\begin{array}{l}\text { Giraldi-Neto } \\
\text { FO et al, } 2012\end{array}$} & 30 & $\mathrm{~F}$ & I & - & - & - & - & + & - & - & $37 / 38$ & Surgical removal \\
\hline & 22 & $\mathrm{~F}$ & I & - & - & - & - & + & + & - & $37 / 38$ & Surgical removal \\
\hline $\begin{array}{l}\text { Boffano } P \text { and } \\
\text { Gallesio } C \\
2009\end{array}$ & 42 & M & $\mathrm{NI}$ & NI & - & NI & NI & + & - & - & $48 / 49$ & Surgical removal \\
\hline $\begin{array}{l}\text { McIntyre } G, \\
1997\end{array}$ & 19 & $\mathrm{~F}$ & I & + & - & + & + & + & - & - & $37 / 38$ & Surgical removal \\
\hline
\end{tabular}


Research, Society and Development, v. 10, n. 6, e15910615506, 2021

(CC BY 4.0) | ISSN 2525-3409 | DOI: http://dx.doi.org/10.33448/rsd-v10i6.15506

\begin{tabular}{|c|c|c|c|c|c|c|c|c|c|c|c|}
\hline $\begin{array}{l}\text { Robinson JA } \\
\text { et al, } 1991\end{array}$ & 25 & M & $\mathrm{I}$ & - & - & - & - & + & - & + & $37 / 38$ e $47 / 48$ \\
\hline \multirow[t]{3}{*}{$\begin{array}{l}\text { Nakamura T, } \\
1991\end{array}$} & 25 & M & II & NI & + & NI & NI & + & + & + & $\begin{array}{c}36 / 37 / 38 \text { e } \\
46 / 47 / 48\end{array}$ \\
\hline & 17 & M & II & NI & + & $\mathrm{NI}$ & NI & + & - & + & $37 / 38$ e $47 / 48$ \\
\hline & 21 & M & I & NI & - & NI & NI & + & + & + & $\begin{array}{c}36 / 37 / 38 \text { e } \\
46 / 47 / 48\end{array}$ \\
\hline $\begin{array}{l}\text { Van Roof RF, } \\
1973\end{array}$ & 31 & M & II & - & - & + & - & + & - & + & $37 / 38$ e $47 / 48$ \\
\hline
\end{tabular}

ASA: American Society of Anesthesiologist physical status; CBCT: cone beam computadorized tomography; PAN: pantomography; NI: not informed. Source: Authors. 


\subsection{Description and analysis}

The articles that reported age ranged from 10 to 56 years of age, with a mean of 29.12, and a median of 27 years. The men had an average of $28 \pm 8$ and, the women, $27 \pm 9$ years. Most of the patients were 30 years or more $(\mathrm{n}=14,43.8 \%)$, however, there was no difference in the prevalence of age groups $(\mathrm{p}=0.540)$ (Table 2). Concerning sex, 14 were male and 19 females, with no significant difference between men and women $(\mathrm{p}=0.627)$.

Table 2. Distribution of data related to Kissing Molars.

\begin{tabular}{|c|c|c|c|}
\hline & $\mathbf{N}$ & $\%$ & p-Value \\
\hline \multicolumn{4}{|l|}{ Sex } \\
\hline Male & 14 & 42.4 & 0.627 \\
\hline Female & 19 & 57.6 & \\
\hline \multicolumn{4}{|l|}{ Mental retardation } \\
\hline Yes & $22 *$ & 81.5 & 0.023 \\
\hline No & 5 & 18.5 & \\
\hline \multicolumn{4}{|l|}{$\mathbf{A S A}$} \\
\hline I & $21 *$ & 91.3 & 0.009 \\
\hline II & 2 & 8.7 & \\
\hline \multicolumn{4}{|l|}{ Age range } \\
\hline $0-19$ & 7 & 21.9 & 0.540 \\
\hline $20-29$ & 11 & 34.4 & \\
\hline 30 years or older & 14 & 43.8 & \\
\hline \multicolumn{4}{|l|}{ Symtomatology } \\
\hline No & 13 & 43.3 & 0.796 \\
\hline Yes & 17 & 56.7 & \\
\hline \multicolumn{4}{|l|}{ Edema } \\
\hline No & 20 & 76.9 & 0.796 \\
\hline Yes & 6 & 23.1 & \\
\hline \multicolumn{4}{|l|}{ Pain } \\
\hline No & 16 & 53.3 & 0.083 \\
\hline Yes & 14 & 46.7 & \\
\hline \multicolumn{4}{|l|}{ Imaging Exam } \\
\hline PAN & 19 & 59.4 & 0.616 \\
\hline $\mathrm{PAN}+\mathrm{CBCT}$ & 13 & 40.6 & \\
\hline \multicolumn{4}{|l|}{ Diagnostic discovery } \\
\hline Cystic Lesion & 3 & 21.4 & 0.235 \\
\hline Hyperplastic Follicle & 9 & 64.3 & \\
\hline Absence Third molar & 2 & 14.3 & \\
\hline \multicolumn{4}{|l|}{ Laterality } \\
\hline Unilateral & 20 & 62.5 & 0.450 \\
\hline Bilateral & 12 & 37.5 & \\
\hline
\end{tabular}


Research, Society and Development, v. 10, n. 6, e15910615506, 2021

(CC BY 4.0) | ISSN 2525-3409 | DOI: http://dx.doi.org/10.33448/rsd-v10i6.15506

\section{First molar associated}

No

Yes

$28^{*}$

$87.5 \quad \mathbf{0 . 0 0 3}$

Second molar associated

No

$25^{*} \quad 78.1$

Yes

78.1

Third molar associated

No

$2 \quad 6.3$

0.000

Yes

$30^{*} \quad 93.8$

Associated Supernumerary

No

$25^{*}$

78.1

0.036

Yes

$7 \quad 21.9$

Treatment

Extraction

Orthodontics

$25 *$

96.2

0.000

Anesthesia

General

$$
4
$$

10

28.6

0.440

Local

71.4

Surgical Technique

Odontosseccion

$4 \quad 19.0$

0.055

Ostectomy + Odontosseccion

$17 \quad 81.0$

Histomatological analysis

Unrealized

2

Normal folicular tissue

$2 \quad 12.5$

0.370

Hiperplastic folicular tissue

$\begin{array}{ll}7 & 43.8\end{array}$

Follicular cyst

$1 \quad 6.3$

Cyst dentigerous

$1 \quad 6.3$

\section{Follow-up}

$\leq 6$ months

$\geq 6$ months

5

31.3

Postoperative complications

$\begin{array}{lccc}\text { No } & 23 * & 88.5 & \mathbf{0 . 0 0 2} \\ \text { Yes } & 3 & 11.5 & \end{array}$

*p<0.05, Fisher's exact test or Pearson's chi-square test. Data expressed as absolute and percentage frequency. Source: Authors.

A significant majority of patients did not present clinically significant changes being classified as ASA I ( $\mathrm{n}=21$, 91.3\%, p=0.009). Only 5 cases had any systemic alteration or associated neurological disorder $(\mathrm{n}=5,18.5 \%, \mathrm{p}=0.023)$ (Menditti, et al., 2015; Nakamura, et al., 1992; Arjona-Amo, et al., 2016). 
Regarding the presence of symptoms associated with KM, most patients had pain in the retromolar region ( $\mathrm{n}=16)$, however, there was no statistically significant difference. Edema was insignificantly present $(\mathrm{n}=20,76.9 \%$, p=0.796), while pain was reported in $14(46.7 \%)$ of patients ( $\mathrm{p}=0.083)$. In 14 cases, KMs were asymptomatic (Table 2).

KMs were diagnosed on routine radiographs in 9 cases (Table 2). Only 4 cases reported Angle's classification (Barros, et al., 2018; Nuño \& Quijada, 2019; Anish, et al., 2015; Cansiz, et al., 2016). In 19 cases (59,4\%) only panoramic radiography (PR) was used for diagnosis and/or surgical planning. In 13 cases $(40,6 \%)$ the authors used PR in conjunction with cone-beam computed tomography (CBCT) (Figure 3).

Figure 3. Panoramic reconstruction of a 35-year-old woman showing the presence of KMs.

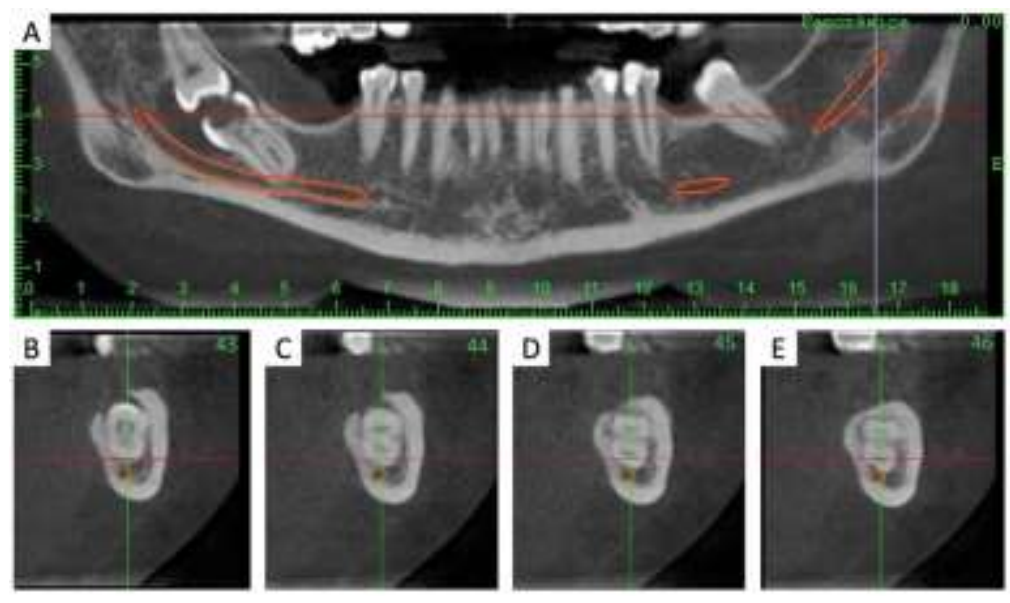

Source: Authors.

As for the teeth involved, they were found to form the combination of KM: the first with the second molar, the second with the third molar, and the third with the fourth molar. There was a greater amount involving the second and third molars unilateral or bilateral $(n=21)$, and a smaller amount involving the first and second unilateral molars $(n=3)($ Table 1). We also found cases of KMs involving the third and fourth molar unilateral $(n=6)$ and bilateral $(n=1)$. An atypical form of KM was verified bilaterally involving the first, second, and third molars (n=2) (Nakamura, et al., 1992).

Cases of bilateral KMs were more prevalent in men than in women $(\mathrm{p}=0.006)$. Regarding the lesion associated with KM, the main diagnostic hypothesis was not reported $(n=19,57.58 \%)$. The thickened follicular tissue $(n=6)$ followed by the dentigerous cyst $(n=2)$ was the most reported diagnostic hypothesis. Nuño \& Quijada (2019), reported a case in which they did not show cystic formation wrapped around the tooth. As for the histopathological diagnosis of these follicles involving the KM crowns (Table 3), 5 cases were dentigerous cysts and 6 cases found thickened pericoronal follicles. No histological diagnosis was shown in more than half of the sample $(n=17,51.51 \%)$. 
Table 3. Correlation of Kissing Molars data with laterality.

\begin{tabular}{|c|c|c|c|c|c|}
\hline & \multicolumn{4}{|c|}{ Laterality } & \\
\hline & \multicolumn{2}{|c|}{ Unilateral } & \multicolumn{2}{|c|}{ Bilateral } & \\
\hline \multicolumn{6}{|l|}{ Sex } \\
\hline Male & 5 & $25.0 \%$ & $9 *$ & $75.0 \%$ & 0.006 \\
\hline Female & $15^{*}$ & $75.0 \%$ & 3 & $25.0 \%$ & \\
\hline \multicolumn{6}{|l|}{ Mental retardation } \\
\hline No & 12 & $85.7 \%$ & 9 & $75.0 \%$ & 0.490 \\
\hline Yes & 2 & $14.3 \%$ & 3 & $25.0 \%$ & \\
\hline \multicolumn{6}{|l|}{$\mathbf{A S A}$} \\
\hline I & 13 & $86.7 \%$ & 7 & $100.0 \%$ & 0.311 \\
\hline II & 2 & $13.3 \%$ & 0 & $0.0 \%$ & \\
\hline \multicolumn{6}{|l|}{ Age } \\
\hline$<30$ & 11 & $55.0 \%$ & 7 & $63.6 \%$ & 0.641 \\
\hline $30 \mid \leq$ & 9 & $45.0 \%$ & 4 & $36.4 \%$ & \\
\hline \multicolumn{6}{|l|}{ Symtomatology } \\
\hline No & 8 & $40.0 \%$ & 4 & $44.4 \%$ & 0.822 \\
\hline Yes & 12 & $60.0 \%$ & 5 & $55.6 \%$ & \\
\hline \multicolumn{6}{|l|}{ Edema } \\
\hline No & 15 & $83.3 \%$ & 5 & $62.5 \%$ & 0.245 \\
\hline Yes & 3 & $16.7 \%$ & 3 & $37.5 \%$ & \\
\hline \multicolumn{6}{|l|}{ Pain } \\
\hline No & 9 & $45.0 \%$ & 6 & $66.7 \%$ & 0.280 \\
\hline Yes & 11 & $55.0 \%$ & 3 & $33.3 \%$ & \\
\hline \multicolumn{6}{|l|}{ Imaging exam } \\
\hline PAN & 11 & $55.0 \%$ & 8 & $66.7 \%$ & 0.515 \\
\hline $\mathrm{PAN}+\mathrm{CBCT}$ & 9 & $45.0 \%$ & 4 & $33.3 \%$ & \\
\hline \multicolumn{6}{|l|}{ Diagnostic discovery } \\
\hline Cystic Lesion & 1 & $12.5 \%$ & 1 & $20.0 \%$ & 0.473 \\
\hline Hyperplastic Follicle & 5 & $62.5 \%$ & 4 & $80.0 \%$ & \\
\hline Absence Third molar & 2 & $25.0 \%$ & 0 & $0.0 \%$ & \\
\hline \multicolumn{6}{|l|}{ First molar associated } \\
\hline No & 18 & $90.0 \%$ & 10 & $83.3 \%$ & 0.581 \\
\hline Yes & 2 & $10.0 \%$ & 2 & $16.7 \%$ & \\
\hline \multicolumn{6}{|l|}{ Second molar associated } \\
\hline No & 6 & $30.0 \%$ & 1 & $8.3 \%$ & 0.151 \\
\hline Yes & 14 & $70.0 \%$ & 11 & $91.7 \%$ & \\
\hline \multicolumn{6}{|l|}{ Third molar associated } \\
\hline No & 2 & $10.0 \%$ & 0 & $0.0 \%$ & 0.258 \\
\hline Yes & 18 & $90.0 \%$ & 12 & $100.0 \%$ & \\
\hline
\end{tabular}




\section{Associated Supernumerary}

No

Yes

\section{Treatment}

Extraction

Orthodontics

\section{Anesthesia}

General

Local

\section{Surgical Technique}

Odontosseccion

Ostectomy + Odontosseccion

\section{Histomatological analysis}

Unrealized

Normal folicular tissue

Hiperplastic folicular tissue

Follicular cyst

Cyst dentigerous

\section{Follow-up}

$$
\leq 6 \text { months }
$$

$\geq 6$ months

\section{Postoperative complications}

\begin{tabular}{lccccc} 
No & 13 & $86.7 \%$ & 9 & $100.0 \%$ & 0.253 \\
Yes & 2 & $13.3 \%$ & 0 & $0.0 \%$ & \\
\hline
\end{tabular}

*p<0.05, Fisher's exact test or Pearson's chi-square test. Data expressed as absolute and percentage frequency. Source: Authors.

The treatment of choice was surgical intervention $(n=25,96,2 \%)$. In all cases, the surgical technique of odontossection was used to remove these teeth $(\mathrm{n}=17,81 \%, \mathrm{p}=0.055)$. Only two cases presented orthodontic treatment as a viable possibility in the management of KMs (Barros, et al., 2018; Ghosh, et al., 2017). There was no difference between anesthetic protocols (general or local) $(\mathrm{p}=0.440)$ and most of the studies did not describe the anesthetic protocol used $(\mathrm{n}=19$, $57.58 \%$ ) (Table 2) (Figure 4).

$\begin{array}{ccccc}14 & 70.0 \% & 11 & 91.7 \% & 0.151 \\ 6 & 30.0 \% & 1 & 8.3 \% & \end{array}$

$18 \quad 94.7 \% \quad 6 \quad 100.0 \% \quad 0.566$

$\begin{array}{llll}1 & 5.3 \% & 0 & 0.0 \%\end{array}$

$\begin{array}{lllll}4 & 40.0 \% & 0 & 0.0 \% & 0.188 \\ 6 & 60.0 \% & 3 & 100.0 \% & \end{array}$

$\begin{array}{cccc}1 & 7.1 \% & 3 * & 50.0 \% \\ 13 * & 92.9 \% & 3 & 50.0 \%\end{array}$

0.028

0.673

$\begin{array}{llll}4 & 66.7 \% & 1 & 50.0 \%\end{array}$


Figure 4. Clinical images of a 35-year-old woman showing the presence of KMs. 4A: teeth involved; 4B: Alveoli after surgical removal.
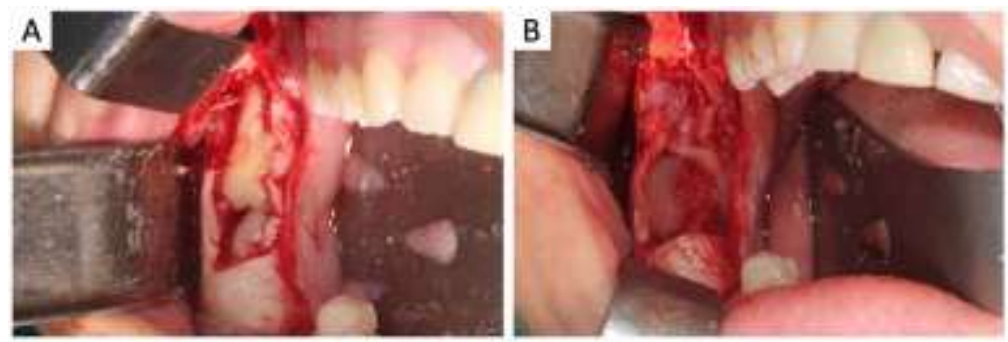

Source: Authors.

Only 9 drug protocols were reported postoperatively among the 33 clinical cases found, of these, 7 used antibiotics associated with non-steroidal anti-inflammatory drugs (Menditti, et al., 2015; Fortes, et al., 2014; Kiran, et al., 2014; ArjonaAmo, et al., 2016; Boffano \& Gallesio, 2009; Nedjat-Shokouhi \& Webb, 2014) and only 1 case associated the analgesic with the previously mentioned drugs (Fortes, et al., 2014).

According to the Gulses, et al (2012) classification for KM, in which the authors report as classification I the association of first and second lower molars with their close contact occlusal, classification II the relationship of second and third molars, and classification III, third and fourth molars. However, the literature describes KM involving first, second, and third molars without specific classification. Thus, a new classification IV would be the simultaneous involvement of three or more molars in close occlusal contact in the posterior mandible, covering $6.1 \%$ of the cases reported in the current literature (Table 4).

Table 4: Kissing Molars classification.

\begin{tabular}{|c|c|}
\hline \multicolumn{2}{|c|}{ Kissing Molars Classification } \\
\hline Classification I & $9,1 \%$ \\
\hline Classification II & $63,6 \%$ \\
\hline Classification III & $21,2 \%$ \\
\hline Classification IV & $6,1 \%$ \\
\hline
\end{tabular}

Source: Authors.

There is no classification in the literature that directs the dental surgeon in choosing the treatment modality. We categorized the KM according to its anteroposterior anatomical location in relation to the ascending branch of the mandible as well as the proximity to the mandibular base that these dental sets meet according to panoramic images or computed tomography (Figure 5). 
Figure 5. Classification of kissing molars (KM) according to its anteroposterior anatomical location concerning the ascending branch of the mandible (line and letters in blue color) as well as the proximity to the mandibular base (line and letters in orange color). XA represents (KM in front of the oblique line of the mandible and distant from the mandibular base); YB represents (KM between the mandibular branch and the body of the mandible and proximity to the mandibular base without contact with the lower mandibular cortex); ZC represents (KM in mostly inserted in the mandibular ramus and close contact with the mandible base).

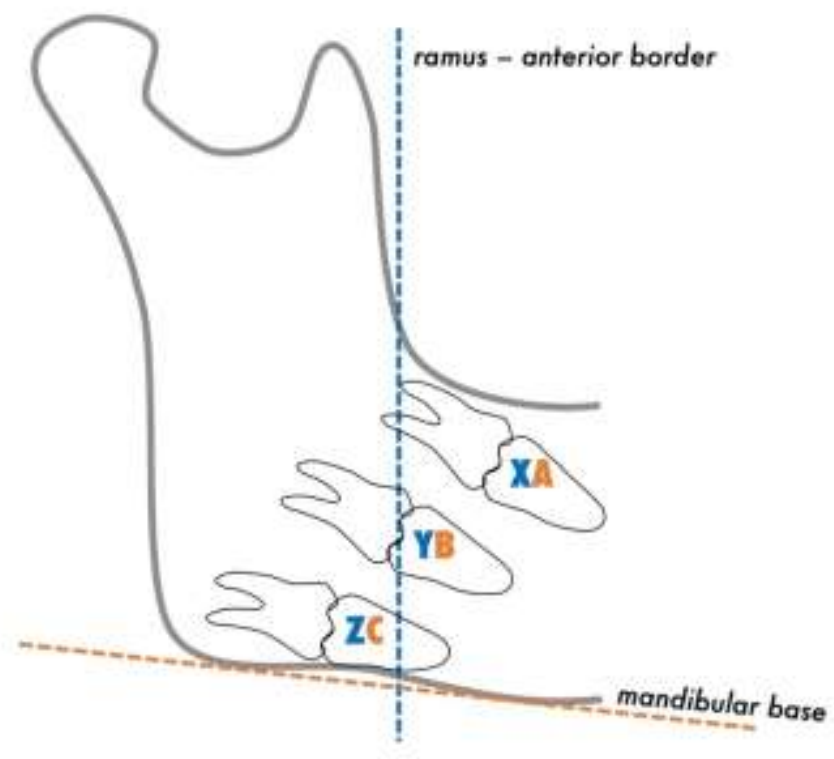

Source: Authors.

\section{Anteroposterior classification}

KMs are closely related to the mandibular ascending branch. When this dental set is in front of the oblique line of the mandible, we can classify them as position X. When the KMs are between the mandibular branch and the body of the mandible, it is named as position $\mathrm{Y}$ and, when mostly inserted in the ascending branch mandibular, it receives the $\mathrm{Z}$ classification in relation to its position. In the literature, 45 dental KM-type sets are reported and $53.3 \%$ of them comprise the $\mathrm{X}$ position in relation to the mandibular ascending branch. 19 cases $(42.2 \%)$ were classified as $\mathrm{Y}$ and two cases were radiographically identified at position $\mathrm{Z}$ (comprising $4.5 \%$ ).

\section{Superoinferior classification}

KMs may also be in proximity with the base of the mandible. Classification A shows a distant relation of the KM to the mandibular base. Position B shows proximity to the mandible base, but there is no contact with the lower mandibular cortex. The classification C involves KM, associated or not with injuries, in close contact with the mandible base, and with increased risk of accidents and operative complications.

Given this classification, the literature reports 17 cases of $\mathrm{KM}$ in position A concerning the mandibular base, comprising $37.8 \%$ of these dental sets. In $28.9 \%$ of cases, position B is observed in 13 cases, and in $33.3 \%$ of cases, position C is observed.

Postoperative complications were absent in most cases ( $\mathrm{n}=23,69.69 \%)$. Cansiz, et al (2016) and Boffano \& Gallesio (2009) reported, respectively, temporary paresthesia of the inferior alveolar nerve, and pulp vitality loss of the tooth adjacent to the KM. McIntyre (1997) reported alveolitis as a complication in his case where a kidnapping had to be removed from the 
Research, Society and Development, v. 10, n. 6, e15910615506, 2021

(CC BY 4.0) | ISSN 2525-3409 | DOI: http://dx.doi.org/10.33448/rsd-v10i6.15506

surgical site. Postoperative follow-up ranged from 10 days to 1 year, however, in 24 studies (72.73\%) follow-up times were not identified.

\subsection{Methodology quality}

For RoB classification, 7 studies (33.3\%) were classified as low risk, 10 (47.6\%) were classified as moderate risk, and 4 $(19.1 \%)$ were classified as high risk, and an overview of the percentage of responses for each item is presented in Figure 6. Regarding the categorization of articles according to the methodological quality based on CARE guideline, the studies were classified as follows: good - 9 studies (42.85\%); reasonable - 7 articles (33.34\%); bad - 4 articles (19.05\%); too bad - 1 article (4.76\%). There was no article classified as excellent, which shows heterogeneity among the studies (Table 5).

Figure 6. Risk of bias graph presents the authors' judgments on each risk of bias item presented as percentages across all included studies.

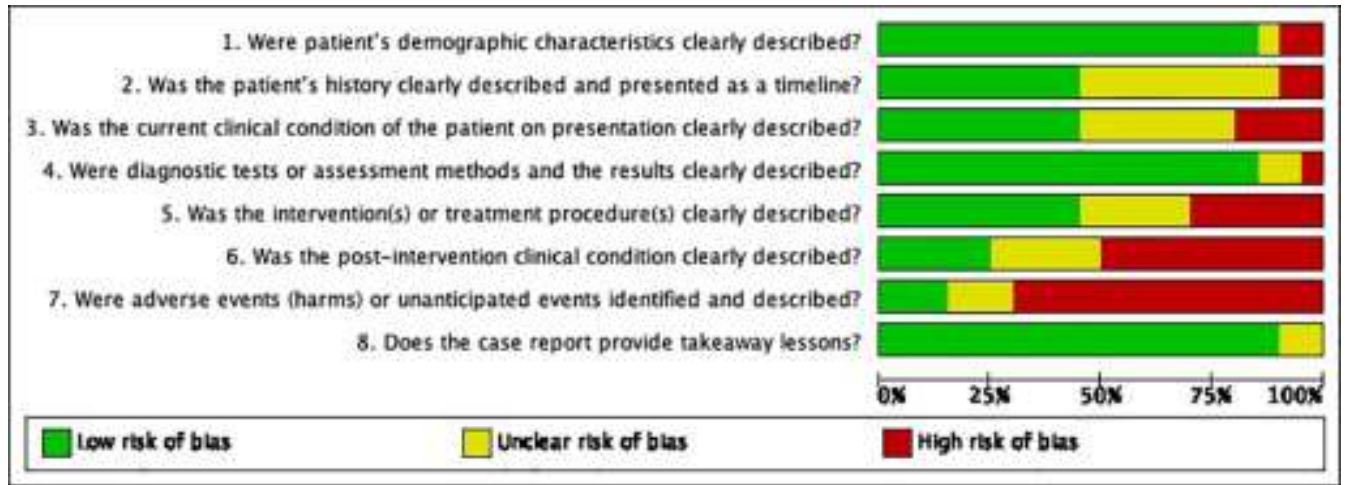

Source: Authors. 
Table 5: Categorization of articles according to the methodological quality based on CARE.

\begin{tabular}{lllllc}
\hline AUTHOR & Yes & No & NA* & \% & Classification \\
\hline Soto and Soto, 2019 & 16 & 14 & 0 & 53.3 & Reasonable \\
Barros et al, 2018 & 21 & 8 & 1 & 70 & Good \\
Bozkurt et al, 2017 & 16 & 13 & 1 & 53.3 & Reasonable \\
Arjona-Amo et al, 2016 & 19 & 9 & 2 & 63.3 & Good \\
Cansiz et al, 2016 & 19 & 9 & 2 & 63.3 & Good \\
Gosh et al, 2016 & 16 & 13 & 1 & 53.3 & Reasonable \\
Zerener et al, 2016 & 20 & 08 & 2 & 66.6 & Good \\
Anish et al, 2015 & 9 & 18 & 3 & 30 & Good \\
Gonzalez-Perez et al, 2015 & 21 & 7 & 2 & 70 & Good \\
Menditti et al, 2015 & 20 & 09 & 1 & 66.66 & Good \\
Jannu et al, 2014 & 22 & 08 & 0 & 73.3 & Good \\
Kiran et al, 2014 & 21 & 7 & 2 & 70 & Good \\
Nedjat-Shokouhi and Webb, 2014 & 23 & 05 & 2 & 76.6 & Reasonable \\
Sá Fortes et al, 2014 & 16 & 12 & 2 & 53.3 & Bad \\
Sheuber and Bornstein, 2014 & 07 & 22 & 1 & 23.3 & Reasonable \\
Giraldi Neto et al, 2012 & 15 & 13 & 2 & 50 & Reasonable \\
Boffano and Gallesio, 2009 & 14 & 13 & 3 & 46.6 & Reasonable \\
McIntyre, 1997 & 18 & 11 & 1 & 60 & Too bad \\
Robinson et al, 1991 & 4 & 23 & 3 & 13.3 & Bad \\
Nakamura et al, 1991 & 12 & 17 & 1 & 40 & Bad \\
Von Roof, 1973 & 7 & 22 & 1 & 23.3 & \\
\hline
\end{tabular}

*NA= Not applicable. Source: Authors.

\section{Discussion}

KMs are rarely reported in the literature and their etiology is still unknown (Zerener, et al., 2016; Robinson, et al., 1991). The largest number of cases reported by region were Italy and Spain, which the latter published by Arjona-Amo, et al. (2016) covers the largest series of cases, including 4 patients with unilateral KM. The prevalence of KM in males is slightly higher, involving patients ranging from 10 to 56 years (Menditti, et al., 2015; Barros, et al., 2018), with a mean age of diagnosis of 28.3 years.

As for the articles selected in this study, only two reported an association of KMs with mucopolysaccharidosis (Van Hoof, 1973; Nakamura, et al., 1992). Cawson (1962) associated the KM dental arrangement with Gargolism. Nakamura, et al. (1992) published a series of four cases in which two patients with KM presented characteristics of this metabolic disorder. The authors conclude that there may be a relationship between KM and mucopolysaccharidosis, but impacted molar molars are not a pathognomonic factor, and RF may exist in patients without systemic alterations as an isolated characteristic. The mucopolysaccharidosis is inherited metabolic disorders, in which there will be abnormal deposition of mucopolysaccharides in the tissues like, skin, soft tissues of airway, cornea, central nervous system, heart, liver, spleen, bones, ligaments, and other sites due to an enzyme abnormality (Shahista, et al., 2013)

The KM mainly involve second and third lower molars, with a higher prevalence of bilateral cases ( $n=9$ ) (Van Hoof, 1973; Zerener, et al., 2016; Robinson, et al., 1991; Fortes, et al., 2014; Anish, et al., 2015; Ghosh, et al., 2017; Jannu, et al., 2014; Kiran, et al., 2014; Nakamura, et al., 1992; Nedjat-Shokouhi \& Webb, 2014) followed by 7 cases involving teeth \#47 and \#48 (Gonzalez-Perez, et al., 2015; Arjona-Amo, et al., 2016; Scheuber \& Bornstein, 2014) and 5 cases of teeth \#37 and \#38 (Fortes, et al., 2014; Ghosh, et al., 2017; McIntyre, 1997; Bozkurt, et al., 2017). In the literature, there is only 1 case of $\mathrm{KM}$ involving teeth \#46 and \#47, described by Barros, et al. (2018) and 1 case involving third and fourth molar quarters, 
repor6ted by Menditti, et al. (2015). The majority of cases found in the literature are bilateral KMs with 12 reports (Van Hoof, 1973; Menditti, et al., 2015; Zerener, et al., 2016; Robinson, et al., 1991; Fortes, et al., 2014; Anish, et al., 2015; Jannu, et al., 2014; Kiran, et al., 2014; Nakamura, et al., 1992; Nedjat-Shokouhi \& Webb, 2014) followed by lefts unilateral KMs (n=11) (Menditti, et al., 2015; Giraldi Neto, et al., 2012; Nuño \& Quijada, 2019; Cansiz, et al., 2016; Arjona-Amo, et al., 2016; McIntyre, 1997; Scheuber \& Bornstein, 2014) and rights unilateral KMs (10 cases) (Barros, et al., 2018; Gonzalez-Perez, et al., 2015; Arjona-Amo, et al., 2016; Boffano \& Gallesio, 2009; Scheuber \& Bornstein, 2014; Bozkurt, et al., 2017). Nakamura, et al. (1992) in the same article, also reported two cases of bilateral KMs involving three teeth, in which one of these patients had type II mucopolysaccharidosis.

The KM may be symptomatic or asymptomatic. The diagnosis, in most reported cases, is motivated by some painful symptomatology (Nuño \& Quijada, 2019; Boffano \& Gallesio, 2009; Scheuber \& Bornstein, 2014), persistent pericoronitis (Robinson, et al., 1991; McIntyre, 1997), or a finding on routine radiographs (Fortes, et al., 2014; Cansiz, et al., 2016; Kiran, et al., 2014; Nedjat-Shokouhi \& Webb, 2014). There have also been reports of edema in the facial region related to KM (Zerener, et al., 2016), and the presence of periodontal pocket in the distal molars adjacent to the KM (Boffano \& Gallesio, 2009).

In the current literature, prophylactic removal of impacted teeth remains a controversial topic. There are some risks in maintaining an impacted tooth even though it is asymptomatic, such as a high incidence of cystic and tumor changes associated with impacted third molars with follicular space less than $2.5 \mathrm{~mm}$, which is considered radiographically normal. Also, as the incidence of systemic diseases, pathological conditions associated with the impacted tooth, and post-surgical morbidity increase with age, so do the difficulties and complications of the impacted third molar surgery. However, some authors support the non-removal of asymptomatic KMs, claiming that the patient will undergo surgical stress without the real indication (Adeyemo, 2006; Eliasson, et al., 1989).

The performance of imaging tests is of great importance for all patients undergoing dental treatment (Van Hoof, 1973). In all cases reported, PR was used for initial evaluation and diagnosis. Some authors used concomitant CBCT and PR for better evaluation (Nakamura, et al., 1992; Nedjat-Shokouhi \& Webb, 2014) and surgical planning (Menditti, et al., 2015; Fortes, et al., 2014; Arjona-Amo, et al., 2016).

The literature is divergent regarding the indication of removal of asymptomatic included third molars. Wu, et al. (2017) in their study involving ectopic third molars, advocated surgical intervention only when clinical symptomatology or when associated with a pathological lesion. Giraldi Neto, et al. (2012) reported two asymptomatic cases of KM surgically removed due to increased follicular space. It justifies the indication due to the increased risk of complications if these teeth were maintained, such as reduced mandibular bone tissue, root resorption of adjacent teeth, pericoronitis, and painful symptomatology.

The primary form of treatment for KMs is surgical removal. However, alternative treatments are also indicated, such as orthodontic traction using extra-alveolar mini-implants and a fixed appliance, as in the study by Barros, et al. (2018).

When treated surgically, complication rates are between 4.6-30.9\% (mandibular fractures during or after surgery, alveolitis, and damage to the inferior alveolar nerve). Barros, et al. (2018) reported that KM should be treated only in cases of clinical symptoms, high risk of caries, or KMs associated with pathologies, periodontal disorders, and progressive bone loss. Nedjat-Shokouhi and Webb (2014) reported conservative treatment when there is no painful symptomatology.

However, Boffano and Gallesio (2009) and Fortes, et al. (2014) described in their studies cases of asymptomatic KM in which the patients were submitted to the surgical treatment after compliance of the patient. Regarding the treatment of choice, it is necessary to observe the patient's main complaint, the individual's age, medical and dental history, associated clinical and imaging findings, dental need, and the patient's free and informed consent for the definition of the best conduct. 
No pattern was found among the authors related to the treatment protocol for cases of kissing molars. Other important factors are highlighted in the choice of the procedure proposed to the patient, such as the patient's economic, social and cultural situation, age of the individual, degree of impaction of the kissing molars, and the affected teeth, team experience and the infrastructure available for the procedure.

In addition to complications associated with the surgical procedure, occlusal sequelae can be generated when surgical removal of the KM is performed, causing an early need for rehabilitation. Thus, Barros, et al. (2018) described a case of KM treated with skeletal anchorage with mini implants for verticalization of this ectopic dental set involving first and second molars with their occlusal in close contact. These teeth were partially erupted and repositioned in the dental arch through extensive root verticalization and with minimal side effects. Other alternative techniques are also described by the authors in their case, which considers conservative and reasonable second molar extraction and orthodontic transposition of the adjacent third molar, as well as second molar extraction and rehabilitation with edentulous area implantation. A careful selection must be made when opting for non-surgical treatments, such as resources and training of the dentist in carrying out the procedure, a patient collaboration concerning oral hygiene and maintenance of the proposed treatment.

As for the surgical techniques, most of the authors opted for the surgical technique with the use of vestibular mucoperiosteal flap posterior region of the mandible, followed by ostectomy and cervical odontossection separating the crown from the root in both molars to extract the KM. Cansiz, et al. (2016) reported a case of KM extraction using the unilateral mandible sagittal osteotomy, proposing an alternative technique for bone preservation and tooth removal. Extractions of kissing molars under general anesthesia are ideal for more complex and invasive cases when there is proximity to the mandibular base, an important impact on the mandibular branch, considering the disposition and anatomy of the teeth to be removed. Non-collaborating patients or patients with systemic disorders, who need auxiliary medical assistance to perform the procedure, are candidates for surgical treatment under general anesthesia when they have an emergency.

Alternative treatments such as KM coronectomy have never been described in the literature. This treatment modality was first proposed in 1984 by Ecuyer and Debien. The technique involves the removal of the dental crown, especially of the lower third molars included when in close contact with the inferior alveolar nerve (IAN), thus aiming to avoid lesions of noble structures adjacent to the dental periapices (Renton, 2013). There are no studies related to coronary angiography in KMs, however, there is an alternative treatment for vital infection-free KMs in close contact with IAN, according to the patient.

Few articles have brought the main diagnostic hypothesis of the lesion associated with KM. The dentigerous cyst and the pericoronary follicular hyperplasia are the most commonly reported in the literature (Menditti, et al., 2015; Fortes, et al., 2014; Anish, et al., 2015; Nedjat-Shokouhi \& Webb, 2014) It is important to emphasize the importance of the histopathological analysis of the lesions associated to KM for the correct clinical outcome. This was observed by Menditti, et al. (2015) in one of their reported cases, in which the diagnosis of the follicular odontogenic cyst was made as a diagnostic hypothesis and the results of the histopathological study were conclusive for dentigerous cyst, with a divergence of the initial diagnosis.

There have been scarcely described drug protocols used after the extraction of KM in their articles. For bacterial control, only antibiotic of the penicillin group has been reported in the literature in combination with anti-inflammatories and analgesics were also prescribed to patients by the same authors (Menditti, et al., 2015; Fortes, et al., 2014; Kiran, et al., 2014; Arjona-Amo, et al., 2016; Boffano \& Gallesio, 2009; Nedjat-Shokouhi \& Webb, 2014).

Regarding postoperative follow-up, most of the authors reported no complications, only Boffano and Gallesio (2009) who reported the loss of tooth pulp vitality adjacent to the surgical site after 60 days. Cansiz, et al. (2016) reported a transient complication after removal of KM, in which the patient evolved with temporary paresthesia of the inferior alveolar nerve with 
recovery in 6 months. McIntyre (1997), in his case, had bilateral alveolitis as a complication in the operated area region in which bone sequestration had to be removed from the tooth cavity.

Despite the limited number of published cases, the occurrence of complications related to the surgical procedure is relatively low. In addition to the patient's response to the performed intervention, KMs in the mandible are usually in close contact with the inferior alveolar nerve, adjacent teeth, near the base of the mandible, and with the presence of cysts and tumors associated with these teeth. These factors increase the risk of complications of these structures. Thus, the importance of careful planning and treatment for the preservation of neighboring structures the KM is emphasized.

\section{Conclusion}

KMs were uncommon findings in the searched literature. Bilateral KMs were statistically more prevalent in males, but the occurrence of KM did not show preference regarding sex and locality. Panoramic radiography was the main imaging exam for KM evaluation. However, CBCT can be an aid to the treatment plan of patients with these dental structural abnormalities. In relation to treatment, careful orthodontic and / or surgical treatments showed acceptable results. Therefore, the importance of accurate planning and careful treatment, with preservation of structures adjacent to the KM for a better clinical outcome should be emphasized.

\section{References}

Adeyemo, W. L. (2006). Do pathologies associated with impacted lower third molars justify prophylactic removal? A critical review of the literature. Oral Surgery, Oral Medicine, Oral Pathology, Oral Radiology, and Endodontology 102(4), 448-52. 10.1016/j.tripleo.2005.08.015

Albuquerque, A. F. M., Sampaio-Melo, C. M., Studart-Soares, E. C., Rodrigues-Ribeiro, T., Roriz-Fonteles, C. S., Alves-Pereira, K. M., Ferreira-Barbosa, D. A., Barros-Silva, P. G., \& Gurgel-Costa, F. W. (2018). Preemptive analgesia-related gene and protein expression in third molar surgeries under non steroidal anti-inflammatory drug protocols: A PROSPERO-registered systematic review of clinical studies. Med Oral Patol Oral Cir Bucal 23:e723-e32. $10.4317 /$ medoral.22576

Anish, N., Vivek, V., Thomas, S., Daniel, V. A., Thomas, J., \& Ranimol, P. (2015). Till Surgery do us Part: Unexpected Bilateral Kissing Molars. Clin Pract 5:688. $10.4081 /$ cp. 2015.688

Arjona-Amo, M., Torres-Carranza, E., Batista-Cruzado, A., Serrera-Figallo, M. A., Crespo-Torres, S., Belmonte-Caro, R., Albisu-Andrade, C., TorresLagares, D., \& Gutiérrez-Pérez, J. L. (2016). Kissing molars extraction: Case series and review of the literature. J Clin Exp Dent 8:e97-e101. $10.4317 /$ jced.52741

Aromataris, E., \& Munn, Z. (2017). Joanna Briggs Institute Reviewer's Manual. https://wiki.jbi.global/display/MANUAL/Chapter+11\% 3A+Scoping+reviews

Barros, S. E., Janson, G., Chiqueto, K., Ferreira, E., \& Rosing, C. (2018). Expanding torque possibilities: A skeletally anchored torqued cantilever for uprighting "kissing molars". Am J Orthod Dentofacial Orthop 153:588-98. 10.1016/j.ajodo.2017.12.006

Boffano, P., \& Gallesio, C. (2009). Kissing molars. J Craniofac Surg 20:1269-70. 10.1097/SCS.0b013e3181abb271

Bozkurt, P., Altindag, A., Ilhan, E., \& Erdem, E. (2017). Kissing molars: report of three cases involving supernumerary tooth, dentigerous cyst and fibroosseous lesion. Cumhuriyet Dent J 20:104-8. 10.7126/cumudj.345891

Cansiz, E., Isler, S. C., \& Gultekin, B. A. (2016). Removal of Deeply Impacted Mandibular Molars by Sagittal Split Osteotomy. Case Rep Dent 2016:1902089. 10.1155/2016/1902089

Cawson, R. A. (1962). The oral changes in gargoylism. Proc R Soc Med 55:1066-70. 10.1177/003591576205501222

Eliasson, S., Heimdahl, A., \& Nordenram, A. (1989). Pathological changes related to long-term impaction of third molars. International Journal of Oral and Maxillofacial Surgery 18(4):210-12. 10.1016/s0901-5027(89)80055-4

Fortes, R. Z. S., Júnior-Salvaro, V., Modolo, F., \& Mackowiecky, E. (2014). Kissing molars: Report of a case. Journal of Oral and Maxillofacial Surgery, Medicine, and Pathology 26:48-51. 10.1016/j.ajoms.2012.09.008

Ghosh, R., Babu, S. G., Castelino, R. L., Nair, M., Rao, K., Bhat, S., \& Banerjee, B. (2017). Kissing molars: a rare case report. Eur J Ther 23:42-4. 10.5152/EurJTher.2017.09120

Giraldi Neto, F. O., Rocha Júnior, H. V., Martinez Júnior, W., Duarte, B. G., Salgueiro, D. G., \& Santana, E. (2012). Interesting cases of kissing molars. Report of two cases. Rev Odontol UNESP 41:292-5. Retrieved from https://www.revodontolunesp.com.br/article/5880192e7f8c9d0a098b500e 
Research, Society and Development, v. 10, n. 6, e15910615506, 2021

(CC BY 4.0) | ISSN 2525-3409 | DOI: http://dx.doi.org/10.33448/rsd-v10i6.15506

Gonzalez-Perez, L. M., Infante-Cossio, P., Sanchez-Sanchez, M., Valdivieso-del-Pueblo, C., \& Robles-Garcia, M. (2015). Kissing Molars: A Report of Three Cases and Literature Review. Int J Oral Dent Health 1:12. 10.23937/2469-5734/1510012

Gulses, A., Varol, A., Sencimen, M., \& Dumlu, A. (2012). A study of impacted love: kissing molars. Oral Health Dent Manag 11:185-8. Retrieved from https://pubmed.ncbi.nlm.nih.gov/23208595/

Jannu, A., Ksn, S. B., Vivek, G. K., Veena, G., \& Kamath, R. (2014). Missing molars caught kissing. IORS J Dent Med Scienc 13:51-4. 10.9790/085313535154

Kiran, H. Y., Bharani, K. S., Kamath, R. A., Manimangalath, G., \& Madhushankar, G. S. (2014). Kissing molars and hyperplastic dental follicles: report of a case and literature review. Chin J Dent Res 17:57-63. Retrieved from https://europepmc.org/article/med/25028691

Liberati, A., Altman, D. G., Tetzlaff, J., Mulrow, C., Gøtzsche, P. C., Loannidis, J. P. A., Clarke, M., Devereaux, P. J., Kleijnen, J., \& Moher, D. (2009). The PRISMA statement for reporting systematic reviews and meta-analyses of studies that evaluate health care interventions: explanation and elaboration. $J$ Clin Epidemiol 62:e1-34. 10.1371/journal.pmed.1000100

McIntyre, G. (1997). Kissing molars: an unexpected finding. Dent Update 24:373-4. Retrieved from https://pubmed.ncbi.nlm.nih.gov/9534425/

Menditti, D., Laino, L., Cicciu, M., Mezzogiorno, A., Perillo, L., Meditti, M., Cervino, G., Lo Muzio, L., \& Baldi, A. (2015). Kissing molars: report of three cases and new prospective on aetiopathogenetic theories. Int J Clin Exp Pathol 8(12):15708-18.

Nakamura, T., Miwa, K., Kanda, S., Nonaka, K., Anan, H., Higash, S., \& Beppu, K. (1992). Rosette formation of impacted molar teeth in mucopolysaccharidoses and related disorders. Dentomaxillofac Radiol 21:45-9. 10.1259/dmfr.21.1.1397452

Nedjat-Shokouhi, B., \& Webb, R. M. (2014). Bilateral kissing molars involving a dentigerous cyst: report of a case and discussion of terminology. Oral Surg 7:107-10. 10.1111/ors.12118

Nuño, A. I. S., \& Quijada, A. S. (2019). Distomolars in ectopic position (kissing molars): report of a case. Revista ADM 76:44-48. Retrieved from https://www.medigraphic.com/cgi-bin/new/resumenI.cgi?IDARTICULO $=85402$

Ouzzani, M., Hammady, H., Fedorowicz, Z., \& Elmagarmid, A. (2016). Rayyan-a web and mobile app for systematic reviews. Syst Rev 5:210. $10.1186 / \mathrm{s} 13643-016-0384-4$

Renton, T. (2013). Update on coronectomy. A safer way to remove high risk mandibular third molars. Dent Update 40(5):362-364, 366-8. $10.12968 /$ denu.2013.40.5.362

Riley, D. S., Barber, M. S., Kienle, G. S., Aronson, J. K., Schoen-Angerer, T. V., Tugwell, P., Kiene, H., Helfand, M., Altman, D. G., Sox, H., Werthmann, P. G., Moher, D., Rison, R. A., Shamseer, L., Koch, C. A., Sun, G. H., Hanaway, P., Sudak, N. L., Kaszkin-Bettag, M., Carpenter, J. E., \& Gagnier, J. J. (2017) CARE guidelines for case reports: explanation and elaboration document. J Clin Epidemiol 89:218-35. 10.1016/j.jclinepi.2017.04.026

Robinson, J. A., Gaffney Jr, W., \& Soni, N. N. (1991). Bilateral 'kissing' molars. Oral Surg Oral Med Oral Pathol 72(6):760. 10.1016/0030-4220(91)90026-9

Scheuber, S., \& Bornstein, M. (2014). Kissing molars. A peculiar radiologic finding. Swiss Dent J 124:16-7. Retrieved from https://www.sso.ch/fileadmin/pubmed/sdj-2014-01-06.pdf

Shahista, P., Mascarenhas, R., Shetty, S., \& Husain, A. (2013). Kissing molars: an unusual unexpected impaction. Archiv Med Health Sci 1:52-3. $10.4103 / 2321-4848.113570$

Tufanaru, C., Munn, Z., Aromataris, E., Campbell, J., \& Hopp, L. (2017). Chapter 3: Systematic reviews of effectiveness. In: Aromataris E, Munn Z (Editors). Joanna Briggs Institute Reviewer's Manual. The Joanna Briggs Institute. Retrieved from https://reviewersmanual.joannabriggs.org/

Van Hoof, R. F. (1973). Four kissing molars. Oral Surg Oral Med Oral Pathol 35:284. 10.1016/0030-4220(73)90297-1

Wu, Y., Song, Y., Huang, R., Hu, J., He, X., Wang, Y., Zhou, G., Sun, C., Jiang, H., Cheng, J., \& Wang, D. (2017). Comprehensive analysis of ectopic mandibular third molar: a rare clinical entity revisited. Head Face Med 13(1):24. 10.1186/s13005-017-0157-x

Zerener, T., Bayar, G. R., Altug, H. A., \& Kiran, S. (2016). Extremely Rare Form of Impaction Bilateral Kissing Molars: Report of a Case and Review of the Literature. Case Rep Dent 2016: 2560792. 10.1155/2016/2560792 